\title{
Chronic Lung and Gastrointestinal Diseases: Take a Broader Perspective
}

\author{
Tiar Oktavian Effendi ${ }^{1}$, Syifa Mustika ${ }^{2 *(D)}$ \\ ${ }^{1}$ Department of Pulmonology and Respiratory Medicine, Faculty of Medicine, Universitas Brawijaya, Malang, Indonesia. \\ ${ }^{2}$ Gastroentero-Hepatology Division, Department of Internal Medicine, Faculty of Medicine, Universitas Brawijaya, Malang, \\ Indonesia.
}

\begin{tabular}{l} 
ARTICLE INFO \\
\hline Article history: \\
Received 28 October 2021 \\
Received in revised form 18 \\
January 2022 \\
Accepted 26 January 2022 \\
Available online 30 January 2022 \\
\hline Keywords: \\
Asthma, \\
COPD, \\
GERD, \\
Human \& medicine, \\
Microbiota.
\end{tabular}

\begin{abstract}
Chronic lung diseases, such as asthma and chronic obstructive pulmonary disease (COPD), are often found with gastrointestinal symptoms or even gastrointestinal diseases as one of its comorbid. Vice versa, many cases of gastrointestinal diseases such as gastroesophageal reflux disease (GERD) have developed respiratory dysfunction later. The connection between these two systems has become interesting lately and has led to several studies to prove the association. Several theories have emerged to explain this association. This includes changes in microbiota, the mucosarelated immune system of both systems, side effects of the therapeutic given, and pathomechanism related to gastrointestinal diseases such as GERD. Many studies try to prove the connection between the microbiota in the respiratory and gastrointestinal system, and changing the abundance in one of the systems can affect another. Both of the systems also have a similar mucosal membrane in their lining. Those membranes have an immune defence called Mucosal-Associated Lymphoid Tissue (MALT). Lymphatic and circulatory systems facilitate the migration between two mucosal, and these interconnections influence each other. Although the side effect of the therapeutic agent in respiratory diseases (such as inhaled corticosteroid, beta-2 agonist, or anticholinergic) is thought to be one of the causative mechanisms, discontinuation of therapy is the second option. Probiotic supplementation to improve microbiota is still not a strong recommendation for management.
\end{abstract}

\section{INTRODUCTION}

Chronic lung diseases, such as asthma and chronic obstructive pulmonary disease (COPD), are often accompanied by gastrointestinal symptoms or even gastrointestinal disorders as one of their comorbidities. Vice versa, gastrointestinal diseases, e.g., inflammatory bowel diseases (IBD) or gastroesophageal reflux disease (GERD), tend to cause an occurrence of respiratory dysfunction and increase the likelihood of respiratory illness. This interesting connection raises the hypothesis that there is a relationship between the lungs and digestive organs. ${ }^{1}$

Asthma is a well-known chronic lung disease which affects $1-18 \%$ of the population in different countries. It could manifest as various symptoms, of which wheezing, shortness of breath, chest tightness and cough, and expiratory flow limitation of varying degrees could be found. Both symptoms and airflow limitation vary in intensity over time. ${ }^{2}$ Gastrointestinal symptoms are also found in asthma. Classic symptoms of GERD, such as heartburn, were found to go from $25-80 \%$ of asthmatic patients. It was also found those with uncontrolled asthma had more severe gastrointestinal symptoms than those with controlled asthma. ${ }^{3,4}$

As with asthma, COPD patients with more severe airflow limitation tend to have more prominent gastrointestinal symptoms. The severity of COPD is measured based on pulmonary function examination by spirometry, according to the Global Initiative for Chronic Obstructive Lung Disease (GOLD) classification. ${ }^{4}$ COPD definition according to GOLD is a "common, preventable and treatable disease that is characterized by persistent respiratory symptoms and airflow limitation

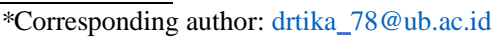

Jurnal Respirasi (Journal of Respirology), p-ISSN: 2407-0831; e-ISSN: 2621-8372.

Accredited No. 200/M/KPT/2020; Available at https://e-journal.unair.ac.id/JR. DOI: 10.20473/jr.v8-I.1.2021.52-59

(c) (1) (2) This work is licensed under a Creative Commons Attribution-Share Alike 4.0 International License. 
that is due to airway and/or alveolar abnormalities usually caused by significant exposure to noxious particles or gases and influenced by host factors including abnormal lung development". Significant comorbidities may affect mortality and morbidity. ${ }^{5}$

With recent studies showing an association between chronic lung disease severity, especially asthma and COPD, and gastrointestinal symptoms, several theories have emerged to explain this association. This includes changes in microbiota, mucosa related immune system, side effects of therapeutic given, and pathomechanism related to gastrointestinal diseases such as GERD.

\section{Change in Microbiota}

This study refers to the microbiome as a collective genome of the microorganism in a specific environment, such as the airway, respiratory tract, and gastrointestinal tract. The community of micro-organisms is called microbiota. Microbiomes encode over 3 million genes and produce thousands of metabolites. Thus, it can replace any host function instead of the human genome, consisting of about 23,000 genes. Furthermore, it can serve as a virtual organ. Change in microbiota consequently influences the host's phenotype, fitness, and health. ${ }^{6}$

Gastrointestinal and respiratory tracts develop from a standard embryonic structure and have similar anatomical structures and mucosal functions. Although the gut and respiratory microbiota might have various compositions, the early-life microbial colonization between the two sites is identical. A study in children with cystic fibrosis showed evidence of a constant crosstalk between intestinal and respiratory microbiota, simultaneously developing after birth. ${ }^{7}$ Micro-aspiration of intestinal micro-organisms is believed to contribute to the development of respiratory microbiota. Fluctuation in the abundance of various bacteria also happens tantamount between two sites. This cross-talk between the gut and lung is usually referred to as the "gut-lung axis". 1,7

Several things can affect the gut microbiota, such as drugs, food ingredients, antibiotics, and feeding practices. ${ }^{1,6}$ Drugs, food consumption, antibiotic use in early life, and feeding practices are also associated with several respiratory diseases. For example, the risk of asthma is highly related to fast food consumption, earlylife acid-suppressive medications and antibiotic intake, caesarian section delivery and formula feeding, while vaginal delivery, breastfeeding and high fibre intake are negatively correlated with asthma. Whether this process is caused by dysbiosis of the gut microbiota, which then affects the respiratory microbiota and causes susceptibility to respiratory diseases, or caused by other mechanisms, is still not proven. ${ }^{1,7}$ Dysbiosis of microbiota in respiratory diseases have already been studied, and it is not only happen in airway respiratory tract but also in the intestinal system. ${ }^{1}$

Early-life exposure to specific microbiota is needed to help the immune system develop and mature. Susceptibility to allergic-related diseases, such as asthma, increases when there is no or less exposure. This study calls it the "hygiene hypothesis" relating to the gutlung axis previously mentioned. Several studies found a compositional difference between gut microbiota of healthy individuals and asthmatic patients, which may be associated with the development of asthma. ${ }^{1}$

Gut microbiota may play a vital role and is associated with COPD development. Cigarette smoking, known as a significant risk factor of COPD, also affects the microbial community and the immune response of the gastrointestinal tract. Only a few studies have identified the direct correlation between these two, thus further studies are needed. ${ }^{1}$ Furthermore, probiotics supplementation like Lactobacillus rhamosus and Bifidobacterium breve showed an anti-inflammatory effect on cigarette smoke-induced inflammation in human macrophages. In animal studies with COPD, the probiotics also showed an attenuating result of alveolar damage and airway inflammation. Individuals with COPD, in a study, were presented with the excellent presentation of Proteobacteria (in particular Haemophilus, Moraxella, and Neisseria spp.) and Firmicutes (Lactobacillus spp.), whereas the amount of Bacteroidetes (specifically, Prevotella spp.) is significantly less. ${ }^{1,7}$

A study of a large demographic lung cancer population proved that exposure to certain antibiotics was correlated with an elevated risk of lung cancer. Antibiotic use was also associated with the alteration of gut microbiota. These two phenomenons indicated a close relationship between lung cancer and gut microbiota. Researchers found a significant difference in gut microbiota beta diversity between healthy and lung cancer individuals using 16s rRNA sequencing. The study had found that patients with lung cancer had an abundance of Enterococcus, despite having fewer phylum Actinobacteria and genus Bifidobacterium. These microbiotas showed as promising biomarkers for lung carcinogenesis. Another study found that gut microbial diversity is also associated with lung cancer therapy. The gut microbial diversity is found higher in subjects with non-small-cell lung cancer (NSCLC) who responded with anti-programmed-death 1 (PD-1) immunotherapy than those who did not. Progression-free survival (PFS) was also associated positively with gut 


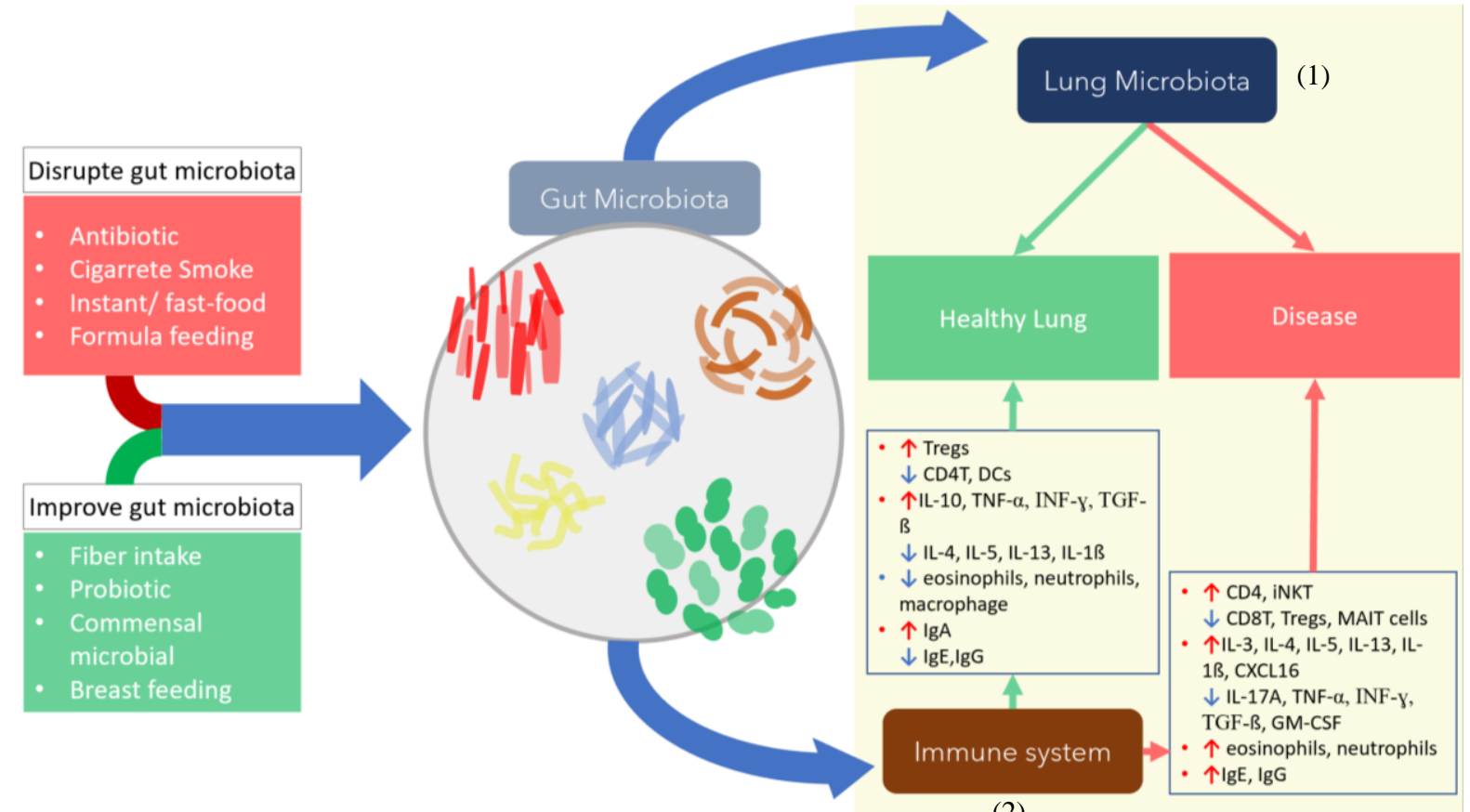

(2)

Figure 1. A possible mechanism of gut microbiota which can influence the respiratory system is through (1) cross-talk between gastrointestinal and lung microbiota and (2) regulation of immune response, both the gastrointestinal system and distal organs.

microbial diversity. Responders of lung cancer therapy showed increased abundances of Akkermansia muciniphila, Eubacterium, Ruminococcus, and Alistipes. They decreased abundances of Parabacteroides and Bifidobacterium in the gut compared with nonresponders. Supplementation of probiotics such as Enterococcus hirae and Barnesiella intestinihominis found to lengthen PFS in mice with advanced lung cancer undergoing chemoimmunotherapy. ${ }^{1}$

A probable mechanism the gut commensal microbiota can influence respiration is through regulation of immune response, both of the gastrointestinal system and the distal organs. The mechanisms include the development of oral immune tolerance through regulatory $\mathrm{T}$ cells (Tregs), the regulation of extraintestinal $\mathrm{T}$ cell populations, production of short-chain fatty acids (SCFA), and regulation of systemic inflammation. The blood and lymphatic system of cytokines and immune cells catalyzed by the gut microbiota and its metabolites, such as SCFAs, can enter the systemic circulation. They will then regulate the inflammatory and immune responses in the lung and further affect respiratory health and diseases (Figure 1). ${ }^{1,6}$

\section{Mucosa-Related Immune System}

A mucosal membrane covers the gastrointestinal, urogenital, and respiratory tracts in their linings. Aside from the skin, the layer is vital as the contact site for exogenous substances and infectious agents. Therefore, a defence mechanism is needed in mucous membranes to protect the body. Several defence mechanisms protect the mucous membrane, including mechanical defences and extensive adaptive and specific immune defences. Immune protection in the mucosal membrane is arranged as a complex of single immune cells, lymphoid tissues, and organs. Mucosa-associated lymphoid tissue or MALT, which refers to all epithelium-associated lymphoid aggregates in the mucosa, are inductive lymphoid structures. Memory and effector cells are generated antigen-primed $\mathrm{B}$, and $\mathrm{T}$ cells are activated for mucosal immunity. ${ }^{8}$

Gut-associated lymphoid tissue (GALT) is referred to MALT within the gastrointestinal tract. The lymphoid tissue of this organ is present in the gastrointestinal tract as diffuse lymphocytic infiltrate or large nonencapsulated aggregations, such as Peyer's patches. The tissue is exposed continually to antigens. It has an essential role in determining the antigens' nature as either harmful (pathogens) or harmless (e.g., food and commensal organism). ${ }^{8}$ The immune system of the intestinal mucosa works in co-adjuvant to the respiratory, immune system. Exposure of the respiratory tract immune system to inhaled antigens should further segregate the bacteria into commensal or potentially catastrophic ones. Therefore, these two immune systems are expected to have comparable potential. MALT in the respiratory and intestinal tract also has morphology and functional characteristics. ${ }^{8,9}$

Animal studies showed that selective trafficking of lymphocytes occurs between these two mucosal surfaces. The lymphatic and circulatory systems facilitate the migration between the two mucosae. This mechanism 
has been proven in numerous animal studies. However, there are only limited data supporting its phenomenon in humans. In humans, B and T lymphocytes, mast cells, and macrophages communicate between the two systems. Activated lymphocytes have been shown to circulate between the mucosal tissues of the lungs, gastrointestinal tract, and salivary glands in asthmatic individuals. ${ }^{9}$

There are several changes in the stomach and small intestine of asthmatics individuals. It includes epithelial gland cell atrophy, crypt's degeneration in the stomach surface, erosive lesion, hyperplasia, and hyperfunctioning of the parietal cell. Hyperplasia of the parietal cell increases hydrochloride acid production and secretion in stomachs, in conjunction with erosive lesions. This hyperfunctioning is thought to be induced by overstimulating histamine by enterochromaffin-like cells (ECL) and mast cells. Hemodynamics alterations are also found in gastric endothelial folds amongst asthmatics. The increase of histamine, IL-8, and vascular endothelial growth factor (VEGF) in asthmatics, which caused angiogenesis, hypertrophy, and bronchial vasodilation, has been identified as possible candidates for these hemodynamic alterations. An increase of IL-13, IL-5, and cellular macrophage colony-stimulating factor (CMSF) found in the mucosa of asthmatics airways is also found in the gastrointestinal tract's lamina propria's eosinophil and mast cells cytoplasmic granules. In any case, it is still unknown whether these eosinophils and mast cells are rising from the asthmatic respiratory framework through the standard mucosal immune system or shows up within the little digestive tract due to conceivable inflammatory stimuli from encompassing regions. ${ }^{9}$

Fibrosis is one of the prominent features shown in the airway remodelling of asthmatic individuals. The secretion of various growth factors, inflammatory molecules, and extracellular matrix elements can affect the structure of the airways. Interestingly, fibrosis in the small intestine is also found in asthmatics. Moderate diffuse fibrosis found in the small intestine occurs in response to inflammation, which could be found present in the gastrointestinal tract of asthmatic individuals due to the increase of leukocyte influx. Furthermore, the inflammatory state can occur, and an increase of gastrointestinal symptoms in asthmatics becomes more prominent. $^{8,9}$

\section{Therapeutical Side Effects}

In chronic lung diseases such as asthma and COPD, inhalation therapy such as bronchodilator and inhaled corticosteroid are the mainstay of treatment. Inhaled corticosteroids (ICS) have been the cornerstone of asthma treatment. Although inhalation therapy has a lower systemic absorption than oral or systemic treatment, some patients may have drug-related adverse effects. The amount of an ICS reaching the systemic circulation is the sum of the drug available after absorption across lung and nasal mucosa, plus the gastrointestinal tract absorption of the swallowed drug. ${ }^{10,11}$ While the target of the inhaled medication is the lung, a considerable amount of inhaled corticosteroid appears in the gastrointestinal tract, including the lining of the oesophagus, the stomach, intestine and colon. About $60 \%$ to $90 \%$ of a dose delivered from a metereddose inhaler (MDI) or dry powder inhaler (DPI) is deposited and subsequently absorbed from the gastrointestinal tract. Pharmacokinetic and pharmacodynamics parameters of ICS, such as systemic clearance rate, the volume of distribution, half-life, accumulation, protein binding property, and first-passage liver metabolism, contribute to overall systemic exposure. Other relevant factors are the glucocorticoid molecule administered, the inhaler device used, the particle size, the aerosol formulations, the dose and amount of drug which is delivered to the lungs and absorbed. ${ }^{10,12}$

Using ICS also increases the risk of oral candidiasis, hoarse voice, easy bruising, and pneumonia even with a low dose. The risk of pneumonia rises in active smokers who are more than 55 years old, have a body mass index lower than 25 , and have severe airflow limitation. ${ }^{5,13}$ Related to the gastrointestinal tract, the inhaled corticosteroid is related to gastritis, ulcer, or bleeding. Approximately $6.4 \%$ of patients using ICS have this side effect related to the dose and duration of the drug used. One retrospective study shows interesting results related to the gastrointestinal side effect of using corticosteroids. Using oral corticosteroids did not significantly increase the risk of gastrointestinal side effects, but ICS did. It was believed that the duration of the corticosteroid used is responsible for its side effects more than how the drug is administered. However, many conflicting results from different studies showed ulcers occurred even within days after oral corticosteroid administration. ${ }^{10,11}$ Only a few studies compare each type of inhaled steroids which are widely available, namely budesonide, fluticasone propionate, beclometasone dipropionate, mometasone furoate, ciclesonide, and flunisolide, with the frequency of its side effect. Fluticasone may have a higher systemic effect compared to budesonide. ${ }^{14}$

The use of beta- 2 agonist is usually related to sinus tachycardia, palpitation, and even severe adverse effects like arrhythmia. Somatic tremor is also a disturbing complaint that happens. ${ }^{5}$ Although gastrointestinal symptoms related to beta-2 agonist 
inhalation are rare, symptoms like nausea or vomiting are reported, especially during high dose administration. A beta- 2 agonist is known to decrease the pressure of the lower oesophagal sphincter (LES), leading to an increase in the likelihood of gastroesophageal reflux in asthmatics. $^{13}$ Similar to beta-2 agonist, the use of antimuscarinic inhalation also has minor effects on the gastrointestinal tracts. Anti-cholinergic is known to decrease gut motility and secretion function of the gastrointestinal tract. In high doses, it can inhibit the secretion of pepsin and mucin. Inhibition of gut motility can affect gastric emptying, which is known to be a risk factor for GERD. ${ }^{5,13}$

\section{Pathomechanism of GERD and Lung Disease}

GERD is a pathologic condition which results from gastric contents into the oesophagus with many symptoms due to the oesophagus, larynx, and respiratory tract involvement. ${ }^{15,16}$ GERD has become a significant problem in many countries, with a global prevalence of around $13 \%$, and continues to increase even $50 \%$ more when compared to the year $1990 .{ }^{4}$ Significant association between GERD and asthma was found with estimation $50 \%$ of asthmatics have GERD symptoms, and almost $80 \%$ asthmatics have abnormal oesophagal $\mathrm{pH}$. Aside from asthma, GERD is also associated with COPD, bronchial nodule granulomatous, bronchiolitis obliterans with organising pneumonia (BOOP), idiopathic pulmonary fibrosis (IPF), and non-tubercular mycobacterial infection. ${ }^{17,18}$ GERD is known to occur in up to $60 \%$ of COPD patients and is associated with an increased risk of exacerbation. The more severe the degree of COPD, the more prominent gastrointestinal symptoms. ${ }^{18,19}$

The reflux was caused by the incompetence of the lower oesophagal sphincter (LES). Reflux can occur through 3 mechanisms: spontaneous reflux during LES relaxation, backflow of gastric content during the swallowing process, and increase of abdominal pressure. ${ }^{15,20}$ Physiologically, LES relaxation only occurs briefly after the swallowing process. Patients with chronic respiratory diseases usually have a higher intraabdominal pressure. That will, in turn, relatively lower intra-thoracal pressure and increase transdiaphragmatic pressure. It is due to increased transdiaphragmatic pressure which leads to more prominent LES incompetence. ${ }^{17,20}$ Also, through an unknown mechanism, a bronchial provocation test with methacholine substances in asthmatics was found to cause bronchospasm and increased the relaxation frequency of LES which caused gastroesophageal reflux..$^{20}$
There are two main mechanisms of how gastroesophageal reflux cause lung disease: (1) a neural reflex came from stimulation of reflux material into the distal oesophagus, and (2) direct effect of reflux material into the proximal oesophagus, larynx, upper respiratory airways, and even to the tracheobronchial tree. ${ }^{20}$ Several studies have demonstrated temporal relation between distal oesophagal reflux and cough using simultaneous ambulatory multichannel intraluminal impedance-pH oesophagal testing and acoustic cough monitors. This evidence supports the association of oesophagal and bronchial reflex mediated by a shared vagal nerve innervation to the brainstem. ${ }^{17,21}$ Stimulation of reflux material to the oesophagus is also known to induce bronchoconstriction through, again, shared vagal nerve innervation. ${ }^{17}$

Microaspiration appears to occur physiologically in up to $50 \%$ of an average healthy individual, particularly during sleep. However, there is an increase in microaspiration in people with asthma, obstructive sleep apnea, and other chronic respiratory diseases. ${ }^{17,20} \mathrm{~A}$ high pepsin detection in broncho-alveolar lavage (BAL) specimens were found in $59 \%$ of asthmatic patients. Pepsin levels alone did not differ based on disease severity or frequency exacerbation. Aside from pepsin, bile and lipid-laden macrophages found in BAL specimens are direct indicators of gastroesophageal reflux to the tracheobronchial tree. Acute pneumonitis can happen due to aspiration of a large volume of acidic fluid, initially with chemical injury followed by an inflammatory phase. ${ }^{17,21}$ A repeated instillation of small amounts of reflux content will then induce a foreign body reaction, which involves cell-mediated immunity. It could further lead to a pathological pattern of nodular granulomas, giant cells, and bronchiolitis with the alveolar organization. ${ }^{17,20}$ Aspiration of bile acids can induce transforming growth factor (TGF)- $\$ 1$ by human epithelial cells. TGF- $\beta$ was highly important in the fibroproliferative process of IPF. This mechanism may thus induce fibroblast proliferation of the disease. Increased concentrations of pepsin and bile acids serve as high prevalence in patients with IPF. Recent studies have suggested that anti-reflux therapies can prolong survival as well as lower the incidence of acute exacerbation inpatient with IPF. ${ }^{20}$

In advancing COPD, significant anatomic and physiologic changes in the chest can affect the competence of esophagogastric junction. Alignment of the crural diaphragm and lower oesophagal sphincter are displaced in advanced COPD caused by hyperinflation and dysfunction of diaphragmatic crural muscle, predisposing to the incompetence of lower oesophagal 


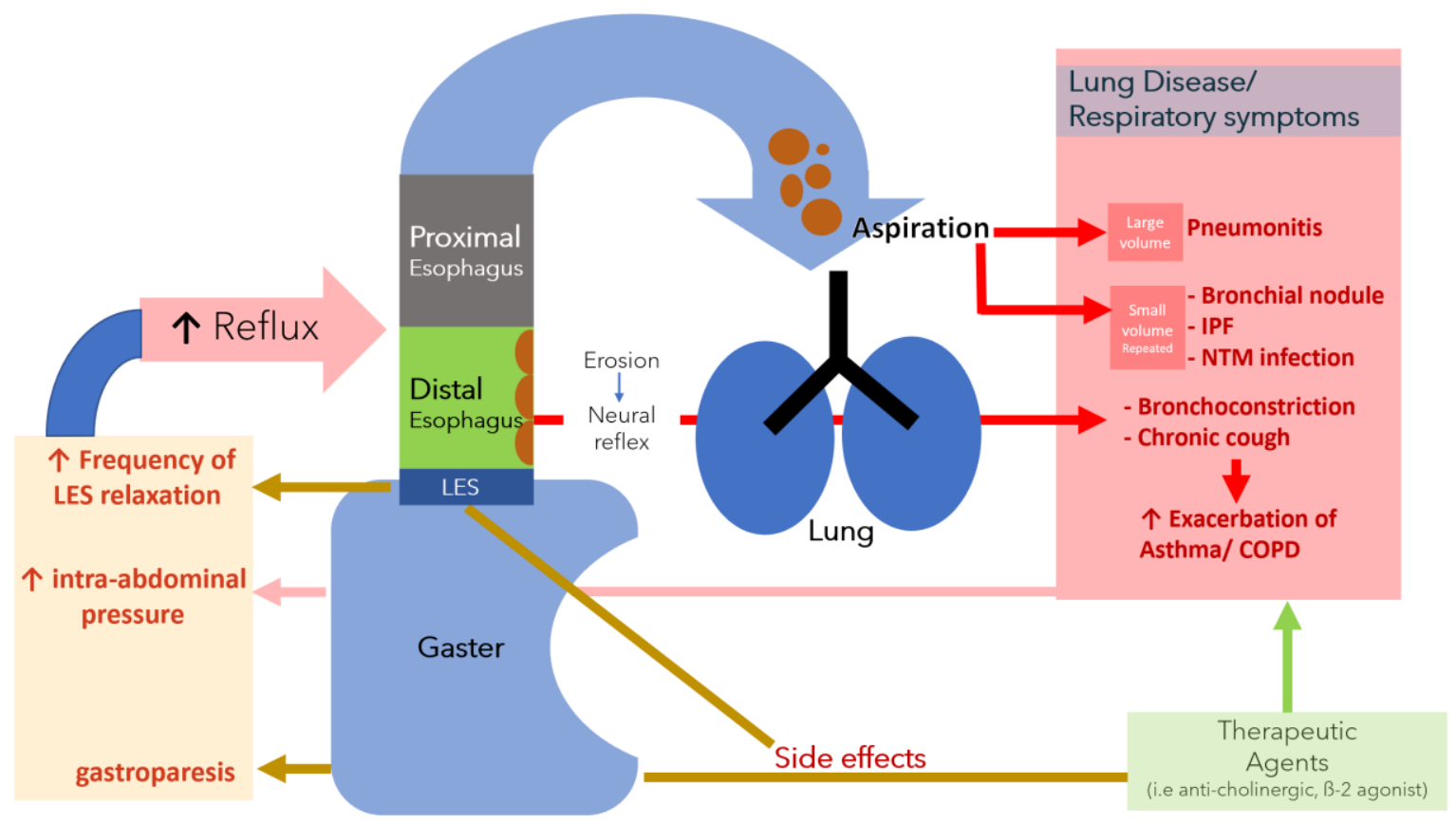

Figure 2. The mechanism by which lung disease can affect the gastrointestinal system and how gastro-oesophageal reflux can cause respiratory problems and lead to diseases. LES: lower oesophagal sphincter; IPF: idiopathic pulmonary fibrosis; NTM: non-tuberculosis mycobacteria

sphincter, and more frequent reflux occurs. ${ }^{17}$ Patients with COPD also show abnormal swallow-breathing coordination, in which the occurrence of swallows are more frequent and in the inappropriate phase of respiration, including during inhalation, and correlates with an elevated risk of aspiration. A summary of this pathomechanism can be seen in Figure 2. ${ }^{22}$

\section{Management of Gastrointestinal Symptoms in Chronic Lung Diseases}

Reflux symptoms in patients with asthma or COPD can be treated with empirical therapy for GERD, using histamine receptor antagonist or proton-pump inhibitor (PPI). PPI is considered superior to histamine receptor antagonists, eliminating preexisting lesions and symptoms up to two times faster. ${ }^{23-25}$ PPI is an $\mathrm{H}^{+}-\mathrm{K}^{+}$ATPase blocker, a final step in gastric acid secretion by parietal cells. Since 1980 when the first generation of PPI was used, PPI has been the most common medication to suppress gastric acid secretion. There are 6 PPIs for clinical use; omeprazole, esomeprazole, lansoprazole, dexlansoprazole, rabeprazole, and pantoprazole. ${ }^{13}$

PPIs are usually given once daily for 8 weeks, can be increased up to twice daily for inpatients with erosive lesions. For inpatient with chronic cough, PPI is recommended to be given twice daily for at least 3 months. Using PPI in asthmatics without reflux symptoms or asymptomatic GERD is still controversial. Empirical therapy of GERD given to those with uncontrolled asthma is no longer recommended. ${ }^{16,23}$
Although it is known that the use of drugs to manage asthma and COPD have side effects related to gastrointestinal symptoms, no consensus or guideline recommends discontinuation of therapy, and optimal treatment is expected to provide maximum therapeutic effect with minimal side effects. However, if the treatment is suspected to be the leading cause of gastrointestinal symptoms, and the complaints continue to persist despite optimal treatment, discontinuing the treatment by switching to another class or route may be considered. ${ }^{2,16}$

Although many studies are researching the use of probiotics in chronic lung disease, such as asthma, COPD, lung cancer, and even IPF, there is still no strong recommendation related to the use of probiotics as part of the management of these diseases. Probiotics supplementation is known to have a preventive effect in children with a high risk of asthma, reduce inflammation in respiratory tract induced by cigarette smokers, and found to prolong PFS in mice with advanced lung cancer undergoing chemoimmunotherapy. Further studies are still needed to assess further the effectiveness and the safety of using probiotics supplementation. ${ }^{1}$

Changes in diet, especially with high fibre intake, are known to reduce the hypersensitivity effect in the lung. ${ }^{3}$ An experimental study on animal models proved that administering a high fermentable fibre diet could protect mice from allergic lung inflammation. There are several possible explanations for this, one of which being a fermentable fibre rich diet will induce the production of 
short-chain fatty acids. In contrast, through a gut-bone axis, this protein would quell allergic reaction in turn by enhancing macrophages and dendritic cell precursors hematopoiesis. This will lead to increased phagocytic activity in the lungs with reduced ability to drive helper T-cell type 2 (Th2) cell effector function., ${ }^{3,26}$ Other possible hypotheses are that a regular, high-fibre-rich diet is assessed to benefit lung function and help reduce COPD risk. Consumption of vegetable and fruits are also known to have a protective feature related to reflux symptoms. ${ }^{26}$

Reducing food consumption which can stimulate acid secretion, such as coffee, chocolate, cheese, and soft drinks, is another thing that can be performed to minimize gastrointestinal symptoms. Avoiding foods which trigger or are suspected to trigger the allergies should be performed. Waiting for two to three hours between last meal and bedtime can reduce the likelihood of heartburn symptoms. ${ }^{16}$

Smoking is a risk factor for lung diseases, such as COPD, and is associated with various symptoms or conditions related to the gastrointestinal tract. Smoking can increase degrade elastin. Elastin is an integral protein found in the respiratory tract and gastrointestinal mucosa. Smoking cessation can reduce not only disease progression but also gastrointestinal symptoms. ${ }^{7,27}$

\section{SUMMARY}

The gastrointestinal and respiratory systems are usually related, whereas gastrointestinal symptoms and vice versa often accompany lung disease. This raises the hypothesis that communication between two systems causes the existing phenomenon. Several theories have been discussed, including changes in the gut and respiratory microbiota, activation of the immune system in the mucosa of the respiratory and gastrointestinal system and its interconnection between them, the side effects of the therapeutics given, and the pathomechanism related to gastrointestinal diseases, such as GERD, in causing lung diseases, and vice versa.

Optimal therapy without discontinuing existing treatment is still the main recommendation despite gastrointestinal symptoms in chronic lung diseases like asthma and COPD. However, if it is thought that therapeutical side effects cause gastrointestinal signs and symptoms to persist despite optimal therapy, a change of drug class and route of administration may be considered. Dietary changes such as providing a high-fibre diet are recommended to reduce gastrointestinal symptoms and improve lung function. Smoking cessation is recommended to minimise worsening of pulmonary function disease progression and lower gastrointestinal symptoms that arise. Although many have researched, probiotic supplementation is still not a strong recommendation for management.

\section{Acknowledgments}

We would like to thank colleagues of Department of Internal Medicine and Department of Pulmonology and Respiratory Medicine, Universitas Brawijaya, for providing all ideas and insight while composing this article.

\section{Conflict of Interest}

The author stated there is no conflict of interest in this study.

\section{Funding}

This study does not receive any funding.

\section{Authors' Contributions}

Writing the manuscript, making figures: TOE. Main idea contributor, reviewing and revising: SM. All authors contributed and have approved the final version.

\section{REFERENCES}

1. Chunxi L, Haiyue L, Yanxia L, et al. The Gut Microbiota and Respiratory Diseases: New Evidence. J Immunol Res 2020; 2020: 2340670. [CrossRef] [PubMed]

2. (GINA) GI for A. Global Strategy for Asthma Management and Prevention (2021 Update). Wisconsin, 2021. [WebPage]

3. Ojha UC, Singh DP, Choudhari OK, et al. Correlation of Severity of Functional Gastrointestinal Disease Symptoms with that of Asthma and Chronic Obstructive Pulmonary Disease: A Multicenter Study. Int J Appl Basic Med Res 2018; 8: 83-88. [CrossRef] [PubMed]

4. Mastronarde JG. Is There a Relationship Between GERD and Asthma? Gastroenterol Hepatol ( $N$ Y) 2012; 8: 401-403. [PMC free article]

5. (GOLD) GI for COLD. Global Strategy for the Diagnosis, Management, and Prevention of Chronic Obstructive Pulmonary Disease (2021 Report). 2021. [WebPage]

6. Valdes AM, Walter J, Segal E, et al. Role of the gut Microbiota in Nutrition and Health. BMJ 2018; 361: k2179. [CrossRef] [PubMed]

7. Marsland BJ, Trompette A, Gollwitzer ES. The Gut-Lung Axis in Respiratory Disease. Ann Am Thorac Soc 2015; 12 Suppl 2: S150-6. [PubMed]

8. Kuper C, Wijnands M, Zander S. MucosaAssociated Lymphoid Tissues. 2017, pp. 81-121.

9. Vieira WA, Pretorius E. The Impact of Asthma on the Gastrointestinal Tract (GIT). J Asthma Allergy 2010; 3: 123-130. [CrossRef] [PubMed] 
10. Heffler E, Madeira LNG, Ferrando M, et al. Inhaled Corticosteroids Safety and Adverse Effects in Patients with Asthma. J Allergy Clin Immunol Pract 2018; 6: 776-781. [CrossRef] [PubMed]

11. Hansen RA, Tu W, Wang J, et al. Risk of Adverse Gastrointestinal Events from Inhaled Corticosteroids. Pharmacotherapy 2008; 28: 13251334. [PubMed]

12. Saag KG, Furst DE, Barnes PJ. Major Side Effects of Inhaled Glucocorticoids. UpToDate, https://www.uptodate.com/contents/major-sideeffects-of-inhaled-glucocorticoids\#H27 (2018, accessed 6 October 2021).

13. Katzung BG. Basic and Clinical Pharmacology. 14th ed. New York: McGraw-Hill Education, 2017.

14. Pandya D, Puttanna A, Balagopal V. Systemic Effects of Inhaled Corticosteroids: An Overview. Open Respir Med J 2014; 8: 59-65. [PubMed]

15. Drupals M, Ware M. Toronto Notes 2021: Comprehensive Medical Reference and a Review for the Medical Council of Canada Qualifying Exam (MCCQE). Toronto, 2021.

16. Alwi I, Salim S, Hidayat R, et al. Penatalaksanaan di Bidang Ilmu Penyakit Dalam: Panduan Praktik Klinis. 4th ed. Jakarta: Pusat Penerbitan Ilmu Penyakit Dalam, 2019.

17. Lee AS, Lee JS, He Z, et al. Reflux-Aspiration in Chronic Lung Disease. Ann Am Thorac Soc 2020; 17: 155-164. [CrossRef] [PubMed]

18. Franssen FME, Rochester CL. Comorbidities in Patients with COPD and Pulmonary Rehabilitation: Do They Matter? Eur Respir Rev 2014; 23: 131141. [PubMed]
19. Sanchez J, Schumann DM, Karakioulaki M, et al. Laryngopharyngeal Reflux in Chronic Obstructive Pulmonary Disease - A Multi-Centre Study. Respir Res 2020; 21: 220. [CrossRef] [PubMed]

20. Morehead RS. Gastro-Oesophageal Reflux Disease and Non-Asthma Lung Disease. Eur Respir Rev 2009; 18: 233-243. [CrossRef] [PubMed]

21. Meyer KC. Gastroesophageal Reflux and Lung Disease. Expert Rev Respir Med 2015; 9: 383-385. [PubMed]

22. Cvejic L, Harding $\mathrm{R}$, Churchward $\mathrm{T}$, et al. Laryngeal Penetration and Aspiration in Individuals with Stable COPD. Respirology 2011; 16: 269-275. [CrossRef]

23. Wang Y-T, Tsai M-C, Wang Y-H, et al. Association between Proton Pump Inhibitors and Asthma: A Population-Based Cohort Study. Front Pharmacol 2020; 11: 607. [PubMed]

24. Trukhmanov AS. Diagnosis and Treatment of Gastroesophageal Reflux Disease. Terapevticheskii Arkhiv 2011; 83: 44-48. [PubMed]

25. Yudhawati R, Parasetiyo YD. Imunopatogenesis Penyakit Paru Obstruktif Kronik. Jurnal Respirasi. 2018; 4(1): 19-25. [CrossReff]

26. Puspita F, Putri L, Rahardja C, et al. Prevalence of Gastroesophageal Reflux Disease and Its Risk Factors in Rural Area. Indones J Gastroenterol Hepatol Dig Endosc 2017; 18: 9. [PubMed]

27. Indonesia PDP. Penyakit Paru Obstruktif Kronik (PPOK): Diagnosis dan Penatalaksanaan. Jakarta: UI Press, 2016. 